\title{
Infusing IVIG through Community Care Access Services in Patients with CIDP
}

\author{
Hans D Katzberg, Vilija Rasutis, Vera Bril
}

Keywords: neuromuscular, neuromuscular disorders, neuropathy, polyneuropathy, therapeutics

Chronic inflammatory demyelinating polyradiculoneuropathy (CIDP) is one of the few neuropathies where immunotherapy can improve strength, sensation, mobility and function. A commonly used treatment strategy for CIDP is use of intravenous immunoglobulin (IVIG), which has the benefit of being well-tolerated in most patients and with evidence of efficacy. ${ }^{1}$ Although this treatment is routinely available as an out-of-hospital infusion in countries such as the United States, England, and Holland, ${ }^{2}$ it is only available for infusion within the hospital setting in Canada.

In an effort to shift point-of-care for IVIG treatment in Canada to a more efficient and cost-saving out-of-hospital model, we performed a proof of principle study of home IVIG infusion in patients with CIDP, published in the Canadian Journal of Neurological Sciences in 2011. ${ }^{3}$ The study showed that infusing IVIG in the home is a feasible and safe option for maintenance therapy in patients with CIDP in Canada. The current study is a follow-up study evaluating the safety and tolerability of maintenance IVIG for patients with CIDP infused in an urban community care access centre (CCAC) service facility where the nursing ratio per patient is higher than the $1: 1$ nursing ratio required for home infusion. Our hypothesis is that treatment of CIDP with maintenance IVIG in the CCAC treatment facilities is feasible, safe and well-tolerated, offering yet another viable option for treatment of chronic neuromuscular conditions outside the hospital setting in Canada.

\section{Materials AND Methods}

The study protocol received ethics approval from the Research Ethics Board of the Institute for the Toronto Community Care Access Program (TOCCAC) in Toronto, and all patients in the study provided consent for this study. Patients with CIDP followed at our neuromuscular clinic at the Toronto General Hospital (TGH) were approached to enter the program, either by phone or during their regular clinic appointments. Inclusion criteria included subjects 18 years of age and older; EFNS confirmed diagnosis of CIDP requiring chronic therapy with IVIG; clinical stability while on treatment in the past six weeks prior to inclusion in the study, and no previous serious adverse reaction from IVIG historically. Exclusion criteria included any other neurological condition(s) interfering with the clinical assessment of CIDP or study assessment, known immunoglobulin A deficiency and female subjects who were pregnant or breastfeeding.

Once patients were deemed eligible for this program, they underwent an initial assessment as part of routine care. Upon meeting eligibility criteria, subjects were admitted into the program. The following assessments were performed upon entry and exit from the program: 1) quality of life index 2) health care utilization questionnaire 3 ) neurological examination including Medical Research Council sum score and sensory testing. All patients enrolled in this program received Gamunex IVIG $1 \mathrm{~g} / \mathrm{kg}$ over one to two days, with pre-treatment medication: diphenhydramine $50 \mathrm{mg}$ and/or acetominophen $650 \mathrm{mg}$ one to two tablets orally, as per their routine/ regular pre-treatment practice and only if pre-treatment was part of their standard pretreatment regimen in the hospital setting. Treatment with IVIG was given as per the patient's usual treatment schedule for a total of three months in four patients and 18 weeks in patients receiving IVIG every 6 weeks. At the completion of the three months of immunoglobulin treatment, patients were asked their level of satisfaction using a patient satisfaction questionnaire with the program, and whether preference was hospital or community based infusion.

Administration of IVIG was divided into the following steps: 1) pick -up and transport of immunoglobulin from Toronto General Hospital 2) preparation and set-up of product 3) patient education 4) administration of immunoglobulin and monitoring/record keeping 5) post-infusion monitoring and surveillance. A neuromuscular nurse with expertise in IVIG infusion (VR): 1) led a program which provided training of procedures used in the Toronto General Hospital medical day unit to CCAC staff 2) initiated and assisted in transfusion of IVIG with patients 3) reviewed all necessary documentation for infusion and follow-up including review of adverse events 4) ensured that administration of immunoglobulin followed the Canadian and American standards for administration of blood products. The CCAC site used for this study is one of four sites in Toronto and one of 14 in Ontario which delivers care to patients which includes wound care and infusion of medications. There are two private patient treatment areas serviced by two to four clinical nurse specialists, and patient throughput ranges from 10 to 30 per day depending on the treatments required. As is the case with hospital-based programs, the prescribing physician (in this case HDK) was responsible for managing any acute IVIG reactions and as such the CCAC team

From the University Health Network; University of Toronto, Division of Neurology, Toronto, Ontario, Canada.

Received June 23, 2015. Final Revisions Submitted August 25, 2015. Correspondence to: Hans D. Katzberg, Toronto General Hospital / University Health Network, 200 Elizabeth Street, 5ES-306, Toronto, Ontario M5G 2C4.

Email: hans.katzberg@utoronto.ca 
had access to the physician's pager with instructions to contact him if this were to occur.

\section{RESUlTS}

Five subjects (four male, one female) ranging in age from 42 to 72 were included in the study. All had been diagnosed with CIDP with symptoms of numbness, tingling and weakness in the extremities (duration of disease 24-48 months) and had previously received IVIG by infusion in the medical day unit of the hospital. Doses were one $\mathrm{g} / \mathrm{kg}$ every four weeks in four patients and every six weeks for patient 5 . During three out of the 15 infusions, two patients were infused simultaneously in the allotted clinic space.

All five patients completed three infusions in the CCAC over a period of 12 weeks in four patients and 18 weeks in the patient receiving IVIG every six weeks. All five patients were able to maintain strength as measured by the Medical Research Council (MRC) sum score (mean 78.6 vs. 75.2 points, $\mathrm{p}=0.45$ ) as did the Jamar hand grip strength (mean 23.4 vs. $28.1 \mathrm{~kg}$ right hand, $\mathrm{p}=0.23$ and mean 21.5 vs. $27.1 \mathrm{~kg}$ left hand, $\mathrm{p}=0.19$ ), and sensation as measured on neurological examination. The SF-36 also showed no significant change in at three months: mean 38.4 vs. 39.6 points, $\mathrm{p}=0.76$ in physical domains (score/100) and 35.7 vs. 35.3 points, $\mathrm{p}=0.89$ in mental domains (score/100).

No serious adverse events were noted and expected, minor adverse events including mild headache was seen in three out of five patients. One patient experienced a re-appearance of facial numbness experienced years prior determined to be due to CIDP; MRI brain was normal without evidence of stroke or intrinsic lesion.

Three of the five patients scored $80 / 80$ in patient satisfaction and two patients scored high at 76/80 and 79/80. Three patients out of five expressed a preference for CCAC infusion compared to hospitalbased infusion, and one patient stated either was acceptable. One of the patients preferred hospital-based infusion because it was closer to his home compared to the CCAC. All subjects expressed satisfaction with the individualized therapy, increased autonomy in management of their own health care, minimization of difficult commutes and time spent in the hospital.

\section{Discussion}

Our results demonstrate that similar to home-based IVIG, out-of-hospital community care based IVIG infusion for neuromuscular conditions in Canada is safe and well-tolerated as maintenance treatment in patients with CIDP. All the patients were highly satisfied and supportive of the community-based IVIG program and most patients preferred infusion in the CCAC service facility to hospital-based treatment.

The notion that administering blood products outside the hospital setting is unsafe has now been proven false through a number of international publications and our own data in the home and community care setting. ${ }^{3,4}$ The priority then is to optimize patient autonomy in order to save visits to hospital, which can be achieved either through infusion of IVIG in the home for patients with significant disability or in a community care access centre where available. Potential benefits from home or CCAC infusion include improved patient autonomy, minimizing time spent away from work, decreased traveling time and costs all have a potential on improving their quality of life.
As in our previous home study, patient satisfaction was high and most patients preferred out-of-hospital infusion to in-hospital treatment. This observation is consistent with experience in the United States and England, where home infusion is a first-line and preferred treatment option for chronic neuromuscular conditions. In Canada's complex geography, access to care for specialized services remains a major limitation to optimal care. The current study demonstrates the feasibility of a safe community care option to supplement current rural outreach programs or the current strained urban hospital-based infusion model. Although the CCAC network is unique to Ontario, there are analogous community programs with similar infrastructure in other provinces in Canada, including community care services in the British Columbia Health Authority and Health and Social Service Centres (CSSSs) in Quebec. Although most other provinces in Canada do deliver out-of-hospital care, this is primarily through in-home programs. To date, no systematic pathway exists for routine administration of IVIG out of hospital through any of these community or home programs, something we hope to change through dissemination of the current results. Studies are also underway to evaluate the efficacy and feasibility of immunoglobulin self-administered subcutaneously, which may also offer an additional out-of-hospital option for patients and clinicians. ${ }^{5}$

There are limitations to our study. Firstly, it is limited to a small group of neuromuscular patients requiring chronic treatment with IVIG, and may not be applicable to all patients requiring IVIG treatment, particularly if they are clinically unstable. The space and nursing aspect constraints throughout different CCAC's across Ontario and across Canada which are not equipped to service patients with infusion-based therapy are also a significant limiting factor. Finally, cost savings thought to be associated with out-of-hospital infusion compared to hospital-based options ${ }^{6}$ remain to be confirmed through formal cost-benefit analysis.

As health care resources continue to be increasingly scarce in Canada, alternative rational and cost-effective treatments and delivery methods are critical, especially as new therapies emerge. We encourage physicians in Canada to continue to consider out-of-hospital treatment programs in the chronic treatment of neuromuscular patients in an effort to improve access, optimize care and shift costly point-of-care treatment from hospital to community treatment for neuromuscular patients.

\section{ACKNOWLEDGMENTS}

This study was funded via an unrestricted educational grant from Grifols Canada Ltd.

\section{Disclosures}

All three authors have received travel support, research funding and honoraria for speaking from Grifols Biotherapeutics and other IVIG manufacturers including CSL Behring:

Hans Katzberg has the following disclosures: Grifols Canada, Advisor, Honoraria and travel support; CSL Behring, Consultant, Speaker, Speaker's fee and travel support; Genzyme Canada, Researcher and speaker, Speaking fees and travel support.

Vilija Rasutis has the following disclosures: Grifols Canada, Advisor, Honoraria and travel support; CSL Behring, Consultant, Speaker, Speaker's fee and travel support. 
Vera Bri has the following disclosures: Grifols, Consultant, Consulting fees; Grifols, Independent Investigator, Grant; CSL, Principal investigator, Grant; CSF, Consultant, Consulting fee.

\section{REFERENCES}

1. Van schaik IN, Winer JB, de Haan R, Vermeulen M. Intravenous immunoglobulin for chronic inflammatory demyelinating polyradiculoneuropathy. Cochrane Database. 2002:Cd 001797.

2. Rigas M, Tandan R, Sterling RJ. Safety of liquid intravenous immunoglobulin for neuroimmunologic disorders in the home setting: a retrospective analysis of 1085 infusions. J Clin Neuromuscul Dis. 2008;10:52-5.
3. Katzberg HD, Rasutis V, Bril V. Home IVIG for CIDP: a focus on patient centred care. Can J Neurol Sci. 2013;40:384-8.

4. Lucas M, Hugh-Jones K, Welby A, Misbah S, Spaeth P, Chapel H. Immunomodulatory therapy to achieve maximum efficacy: doses, monitoring, compliance, and self-infusion at home. J Clin Immunol. 2010;30(suppl 1):S84-9.

5. Markvardsen LH, Debost JC, Harbo T, et al. Subcutaneous immunoglobulin preserves muscle strength in chronic inflammatory demyelinating polyneuropathy. Eur J Neurol. 2013;2: 836-42.

6. Membe SK, Ho C, Cimon K, Morrison A, Kanani A, Roifman CM. Economic assessment of different modalities of immunoglobulin replacement therapy. Immunol Allergy Clin N Am. 2008;2: 861-74. 